EDUCATION

Research, Inovortion and Solutions on-line ${ }^{\circledR}$
PSYCHOLOGY

$1+D+i$
Electronic Journal of Research

in Educational Psychology

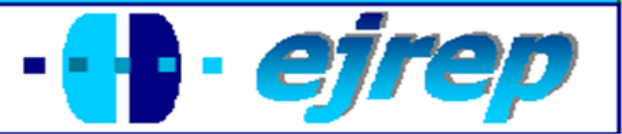

Editorial EOS

\title{
Estudio empírico de variables presagio en la enseñanza-aprendizaje de la estadística a la luz de la investigación sobre competencias*
}

\section{Clemente Rodríguez Sabiote, José Gutiérrez Pérez, Teresa Pozo Llorente}

Facultad de Ciencias de la Educación, Universidad de Granada

\section{España}

Correspondencia: Clemente Rodríguez Sabiote. Departamento de Métodos de Investigación y Diagnóstico en Educación. Facultad de Ciencias de la Educación. Campus de Cartuja, s/n. 18071 Granada. España. E-mail: clerosa@ugr.es

* Trabajo financiado por el Ministerio de Ciencia e Innovación, con cargo al Proyecto ED2008 — 03098.

(C) Education \& Psychology $\mathrm{I}+\mathrm{D}+\mathrm{i}$ and Editorial EOS (Spain) 


\section{Resumen}

Introducción. En la presente investigación pretendemos determinar la influencia que una colección de variables de tipo pronóstico y proceso (opinión del alumnado hacia la estadística, su dedicación a los contenidos de estadística, la valoración de los materiales didácticos y la labor del docente en la enseñanza de la estadística) han jugado en la resolución de actividades y adquisición de competencias relacionadas con el análisis de datos cuantitativos (variable producto).

Método. Se ha desarrollado un estudio correlacional-predictivo con una muestra que corresponde a una cohorte de estudiantes de la Licenciatura de Pedagogía de la Universidad de Granada $(\mathrm{N}=70)$ que se sometieron a un protocolo de cinco situaciones problemáticas y que, además, valoraron con anterioridad la influencia de cinco variables predictivas en la resolución de dichas situaciones.

Resultados. Los resultados alcanzados tras la implementación de un análisis de regresión lineal múltiple indican que, tanto la labor del profesorado, como la actitud hacia la estadística (por este orden) juegan un papel primordial en el rendimiento logrado en la resolución de problemas de estadística y el dominio de competencias de análisis de datos cuantitativos.

Conclusión. El modelo inferido, en este caso particular, ha restado relevancia a la influencia que puedan jugar en el rendimiento estadístico la preparación del alumnado, así como los materiales curriculares puestos a su disposición para su preparación de la asignatura.

Palabras Clave: Rendimiento estadístico, Modelo 3P de Biggs, Modelo DIDEPRO de De la Fuente, Variables determinantes del rendimiento estadístico, Regresión lineal múltiple.

Recibido: 10/11/09 Aceptación Inicial: 19/11/09 Aceptación Definitiva: 11/01/10 


\title{
An empirical study of presage variables on the teaching- learning of statistics in the light of contemporary research on competencies
}

\begin{abstract}
Introduction. In this research we try to determine the influence that a set of presage and process variables (opinion of the students towards the statistics, their dedication to the part of statistics of the subject, teacher's work in the teaching process of the statistical part of the subject ...) they have played in the resolution of activities related to the quantitative data analysis and developed by the students of first year of the Course of Pedagogy of the University of Granada (product variable).

Method. We have developed a correlational-predictive study with a sample that corresponds to students of the Licentiate of Pedagogy of the University of Granada $(\mathrm{N}=70)$. These students have answered a test of five problematic situations and have valued, in addition, five predictive variables related to the resolution of these situations.

Results. The results obtained after the implementation of an analysis of linear multiple regression indicate that the teacher's labour and the attitude towards the statistics (for this order) they play a main role in the performance achieved in the resolution of problems of statistics.

Discussion or Conclusion: The inferred model has reduced importance, as dedication of the students, as the curricular materials put his disposition for the preparation of the subject.
\end{abstract}

Keywords. Statistical achievement, Biggs' 3P model, De la Fuente' DIDEPRO model, influential variables in the statistical achievement, linear multiple regression.

Received: 11/10/09 Initial Acceptance: 19/10/09 Definitive Acceptance: 01/11/10 


\section{Introducción}

La enseñanza de la Estadística posee una importancia destacada en bastantes titulaciones universitarias y su dominio es imprescindible en diferentes áreas de conocimiento, no sólo de las denominadas ciencias "duras", sino también en ciencias sociales, jurídicas y de la salud. Titulaciones como Pedagogía, Psicología y Sociología disponen de materias de carácter obligatorio que permiten a los estudiantes adquirir competencias básicas en su perfil profesional correspondiente. También se da una cierta presencia de la estadística en los currícula de la enseñanza obligatoria, aunque la mayoría de las veces sea de manera básica y se aborde al final del temario, en función de la disponibilidad de tiempo. Sin embargo, como argumentan Behar y Grima (2001), se ha generado un cierto olvido, llamémosle desinterés, por la calidad de los procesos de enseñanza-aprendizaje de dicho tópico. Una de las consecuencias inmediatas ha consistido en la estigmatización de dicha disciplina, no sólo entre el alumnado al que se le imparte, sino además entre algún sector del profesorado que la instruye.

En definitiva, la Estadística como disciplina no despierta, desde luego, grandes simpatías entre el alumnado al que se imparte como norma general. Antes al revés, suele conformar una especie de fobia que Dillon (1982) ha llegado a denominar statisticophia en la que el alumnado percibe la disciplina estadística como un obstáculo para la consecución de su titulación (Perney y Ravid, 1990) y se debate entre sus miedos, ansiedad y actitud poco favorecedora para tratar de aprender y disfrutar de dicha disciplina (Mondejar, Vargas y Mondéjar 2008; Onwuegbuzie y Wilson, 2003; Justicia, Pichardo, Cano, García, De la Fuente, 2008).

Desde estas coordenadas, es importante, por tanto, tratar de establecer mecanismos de regulación de la calidad de los procesos de enseñanza-aprendizaje de la estadística. Una estrategia previa, en este sentido, es considerar una colección de dimensiones o variables que pueden incidir positiva o negativamente en la impartición de contenidos estadísticos en el nivel universitario (Tempelaar, Van der Loeff y Gijselaers, 2007). Con ese objetivo diseccionamos brevemente algunas de estas dimensiones influyentes, tratando de valorar su capacidad predictiva en el desarrollo de buenas prácticas para la Enseñanza-Aprendizaje de la Estadística a la luz de las competencias requeridas en el Espacio Europeo de Educación Superior y los avances de la investigación contemporánea con modelos integradores que incorporan variables de presagio, proceso y producto. 
Marcos de fundamentación y enfoques contemporáneos de la investigación sobre la enseñanza-aprendizaje en la Educación Superior

La construcción de modelos globales para el entendimiento y mejora de los procesos de enseñanza-aprendizaje desde la investigación empírica constituye un campo vigoroso con amplios aportes de investigación a lo largo de todo el siglo XX y que hoy sigue activo. Varias corrientes de investigadores han hecho aportaciones que permiten organizar los hallazgos en, al menos, cinco grandes grupos de influencia. Cada uno de estos grupos inicia su trayectoria histórica en el tiempo con preocupaciones diferenciadas y centros de interés de distinta naturaleza, aunque no resulta fácil establecer límites estrictos de demarcación entre ellos ni entre los tópicos que permitan explicar su sucesión y evolución en el tiempo. Dichos grupos han sido caracterizados en la literatura con las siguientes etiquetas: el paradigma presagioproducto, el paradigma proceso-producto, el paradigma mediacional centrado en el alumnado, el paradigma mediacional centrado en la toma de decisiones del profesor y el paradigma ecológico.

"Las investigaciones apoyadas en ellos se han producido y se están produciendo de forma paralela. Y ni siquiera hoy podemos afirmar que alguno de estos paradigmas se haya instalado definitivamente como el paradigma triunfante. La ciencia de la enseñanza se encuentra en estadios anteriores a la configuración de teorías y consolidación de paradigmas... Sin embargo, ya es posible afirmar que en la actualidad el paradigma presagio-producto no se encuentra vigente y que el paradigma proceso-producto ha recibido tantas críticas y ha tenido que producir tantas explicaciones "ad hoc" y reformularse tan frecuentemente que aparece también como un paradigma agotado o irreconocible” (Pérez, 1983, p. 98).

Trabajos más recientes orientados por marcos de fundamentación como la fenomenografía, el constructivismo o los estudios sobre el procesamiento de la información, tanto desde perspectivas cuantitativas, como cualitativas enfatizan la necesidad de afrontar los problemas de la enseñanza-aprendizaje desde modelos integradores que contemplan la enseñanzaaprendizaje en su contexto, desde las exigencias y presiones institucionales en que se desarrolla y bajo el prisma de las características y percepciones de los agentes involucrados en los procesos. Esto ha obligado a los investigadores a reorientar sus estudios hacia el análisis de: 1) las variables cognitivas, motivacionales y académicas intervinientes (Evans et al. 2003; 
Waugh, 2002; De la Fuente, y Justicia, 2007); 2) la consideración de las preferencias individuales (Hernández, Martínez, Fonseca y Rubio, 2005; Mondéjar et al., 2008; Tempelaar et al., 2007) la apreciación y diferenciación de tipologías de estilos de aprendizaje condicionados por las diferencias contextuales; 4) la incidencia de los materiales curriculares, los recursos virtuales y las presiones del cambio social y la innovación tecnológica; 5) el desarrollo profesional continuado y la necesidad de cualificación permanente que demandan los cambiantes escenarios del mundo laboral.

Esta investigación recupera las aportaciones de una tradición investigadora que requiere de nuevas aproximaciones a la luz de los progresos contemporáneos en el enfoque de la enseñanza-aprendizaje desde la incidencia de nuevas variables de contexto relevantes. Los nuevos modelos de trabajo por competencias que impone la convergencia europea obligan al profesor a establecer controles más sistemáticos de su actividad docente, de los materiales empleados, de las distintas modalidades de clases impartidas, del tiempo requerido para cada actividad, de la pertinencia y adecuación de las mismas al nivel de progresión y avance en los contenidos, de la disponibilidad de prerrequisitos para su ejecución, etc. (De Miguel, 2006).

El trabajo que aquí presentamos trata de ajustarse a estos nuevos enfoques integradores de la investigación en el aula universitaria, recuperando algunos principios relevantes del paradigma presagio-producto; y enfatizando algunos de estos aspectos como nuevos centros de interés que requieren una exploración sistemática continuada en el tiempo en aras de ajustar con fundamento las decisiones didácticas a los nuevos requerimientos de la formación por competencias. Por otra parte, la presencia de la estadística en muchas de las esferas de nuestra vida cotidiana y de las actividades del desempeño profesional justifican su inclusión en los nuevos planes de estudio del título de grado de Ciencias de la Educación, junto a la necesidad de desarrollar una planificación de su enseñanza fundamentada en la investigación contextualizada a la luz de los hallazgos en los estudios sobre el campo.

La exploración de este tema como línea de de investigación pertinente encuentra también su justificación, por una parte, en el rechazo y aversión que suscita en los estudiantes, en los mitos, estereotipos y fobias que predominan sobre la materia; y por otra, en la creciente demanda de su dominio cualificado en los diferentes contextos profesionales. Dentro de la dinámica formativa del EEES, el proceso comienza con la descripción de los resultados de aprendizaje en términos de conocimientos, habilidades, capacidades, comprensión,... que el 
estudiante será capaz de hacer al concluir de manera satisfactoria el proceso formativo. Resultados que se describirán a partir de concebir el currículum como perfiles profesionales integrados por competencias de acción, creando un clima y una atmósfera positiva que haga posible la relación interpersonal y la empatía con el contenido disciplinar así como entre los agentes implicados (profesor-estudiantes), a través de actividades y tareas de enseñanza y evaluación que permitan usar los conocimientos de forma activa y crítica (Hernández et al., 2005: 28; De la Fuente, y Justicia, 2007).

\section{Modelos de nueva generación que integran variables Presagio, Proceso y Producto}

El modelo 3P de Biggs (2005) parte del presupuesto inicial de que los estudiantes utilizan unas u otras estrategias de aprendizaje en función de los motivos que tienen para aprender, siendo éste uno de los núcleos condicionantes del proceso de enseñanza-aprendizaje. Bajo esta estructura, los estudiantes emprenden acciones y realizan tareas en función de aquello que piensan contribuirá a un mayor éxito académico y será recompensado por el sistema en función de los motivos que impulsan su aprendizaje. Esto exige por una parte al profesor ser consciente de que cada estudiante emprende caminos diferentes en su proceso de aprendizaje, y que estos caminos guardan una estrecha relación con las razones que les mueven a encontrar soluciones exitosas.

El modelo DIDEPRO de regulación de la enseñanza-aprendizaje propuesto por De la Fuente y Justicia (2007) muestra como la integración de los elementos contextuales y de los procesos de eseñanza aprendizaje contribuyen de forma eficaz a la autorregulación de los aprendizajes por parte de los estudiantes. Los estudios fenomenográficos sobre los modos de procesamiento de la información relacionados con los distintos estilos cognitivos y las motivaciones estudiantiles que arrojan los diferentes enfoques del aprendizaje en estudiantes universitarios, han sido esquematizados en la literatura al uso en dos grandes grupos amparados en concepciones diferentes sobre el uso del conocimiento: el primer grupo ha sido caracterizado por un enfoque del aprendizaje orientado hacia la actividad memorística y la reproducción superficial de los contenidos objeto de estudio; el segundo, catalogado bajo la etiqueta de aprendizaje profundo es aquel que desarrollan los estudiantes que orientan su aprendizaje hacia metas intrínsecas del conocimiento más reflexivo, activo, comprensivo, etc. 
Los enfoques de enseñanza-aprendizaje profundo y superficial, aún mostrando cierta estabilidad en el tiempo, están sujetos a modificaciones permanentes, fruto de los procesos de adaptación y ajuste entre los agentes y sus entornos; sufren modificaciones y cambios determinados por el contexto en que se desarrollan los aprendizajes y las interacciones interpersonales. Por esta razón, se parte de la idea de que es posible construir y favorecer la creación de un enfoque profundo de la enseñanza-aprendizaje mediante procesos conscientes de planificación de la enseñanza. En nuestro caso, la Estadística y los múltiples mitos que su enseñanzaaprendizaje lleva asociados constituye el foco central de nuestro interés en este artículo. Un análisis más detallado de lo que implica uno u otro tipo de enseñanza-aprendizaje para el profesor y para los estudiantes se presenta a continuación (tablas 1 y 2):

Tabla 1. Enfoque superficial en la Enseñanza-Aprendizaje de la Estadística: factores implícitos para los profesores y estudiantes (Adaptado de Biggs, 2005)

\begin{tabular}{|c|c|}
\hline $\begin{array}{c}\text { Implicaciones del Enfoque Superficial } \\
\text { para el Profesor de Estadística }\end{array}$ & $\begin{array}{l}\text { Implicaciones del Enfoque Superficial } \\
\text { para el Estudiante de Estadística }\end{array}$ \\
\hline $\begin{array}{l}\text { - Enseñar de manera poco sistemática. } \\
\text { - Facilitar listas sin presentar la estructura intrínseca de los } \\
\text { contenidos, ni su relación con el tema o la asignatura. } \\
\text { - Evaluar datos independientes, como se hace con frecuencia } \\
\text { cuando se utilizan respuestas cortas y tests de opción múlti- } \\
\text { ple. } \\
\text { - Presentar poco interés por la materia impartida. } \\
\text { - Dejar tiempo insuficiente para dedicarse de lleno a la tarea, } \\
\text { entizando la cobertura del programa a expensas de la profun- } \\
\text { didad. } \\
\text { - Provocar una ansiedad indebida o unas expectativas restrin- } \\
\text { gidas de éxito frustrantes "el no entienda allá él" }\end{array}$ & $\begin{array}{l}\text { - Persiguen lograr sólo un aprobado raspado, derivado de una } \\
\text { idea de la universidad como una tarea impuesta, obligada, } \\
\text { como una prueba sin sentido de la vida académica que hay } \\
\text { superar de la manera que sea. } \\
\text { - Existen prioridades e intereses extraacadémicos que están por } \\
\text { encima de los académicos. } \\
\text { - Sensación de sobrecarga de trabajo y búsqueda de regateo en } \\
\text { las tareas para reducir tiempos de dedicación. } \\
\text { - Es frecuente mal interpretar las actividades y tareas que se } \\
\text { piden, así como enfocar de manera errónea los esfuerzos a } \\
\text { recordar anécdotas, datos aislados y hechos desconectados. } \\
\text { - Visión escéptica de la educación en permanente cuestiona- } \\
\text { miento. } \\
\text { - Altos niveles de ansiedad. } \\
\text { - Incapacidad para dar pruebas de comprensión del conoci- } \\
\text { miento a niveles de complejidad y argumentando matices. }\end{array}$ \\
\hline
\end{tabular}

Tabla 2. Enfoque profundo en la Enseñanza-Aprendizaje de la Estadística: factores implícitos para los profesores y estudiantes (Adaptado de Biggs, 2005)

\begin{tabular}{|c|c|}
\hline $\begin{array}{c}\text { Implicaciones del Enfoque Profundo } \\
\text { para el Profesor de Estadística }\end{array}$ & $\begin{array}{c}\text { Implicaciones del Enfoque Profundo } \\
\text { para el Estudiante de Estadística }\end{array}$ \\
\hline $\begin{array}{l}\text { Enseñar de manera que se muestre claramente la estructura } \\
\text { de los temas y las relaciones entre los aspectos conceptuales. } \\
\text { Enseñar para suscitar una respuesta positiva por parte de los } \\
\text { estudiantes, formulando preguntas significativas y planteando } \\
\text { problemas relevantes, más que ofreciendo simple informa- } \\
\text { ción. } \\
\text { - Enseñar sobre la base de lo que los estudiantes ya conocen, } \\
\text { partiendo de sus ideas iniciales. } \\
\text { - Cuestionar y transformar las concepciones erróneas de los } \\
\text { estudiantes, considerando el error como fuente de aprendiza- } \\
\text { je. } \\
\text { Evaluar yendo al fondo de los aprendizajes, no a la forma y a } \\
\text { los datos anecdóticos y desconectados. }\end{array}$ & $\begin{array}{l}\text { - Intención de abordar la tarea de manera significativa y ade- } \\
\text { cuada, que puede deberse a una curiosidad intrínseca o a la } \\
\text { determinación de hacer las cosas bien por principios } \\
\text { - Bagaje apropiado de conocimientos, lo cual se traduce en una } \\
\text { capacidad de centrarse en un nivel conceptual elevado y el } \\
\text { despliegue de estrategias de cognición de alto nivel. } \\
\text { - Base de conocimientos bien estructurados asentada sobre } \\
\text { conocimientos previos relevantes y significativos. } \\
\text { - Preferencia auténtica y correspondiente capacidad de respon- } \\
\text { der a cuestiones imprevistas, abiertas, que suponen un desafío } \\
\text { intelectual y buscan la conexión con soluciones complejas. }\end{array}$ \\
\hline
\end{tabular}


Los modelos 3P y DIDEPRO señalas tres puntos temporales en los que se sitúan los factores relacionados con el aprendizaje: pronóstico, antes de que se produzca el aprendizaje; proceso, durante el aprendizaje y producto o resultado del aprendizaje. Las propuestas de Biggs (2005:38) y De la Fuente y Justicia (2007) integran en sus modelos variables PresagioProceso-Producto, como modelos de nueva generación que recuperan la importancia de variables de entrada junto a las mediaciones de proceso, a saber: 1) Las variables presagio engloban variables relacionadas con el contexto de enseñanza tales como contenidos curriculares, método de enseñanza, materiales curriculares, nuevas tecnologías, actuación del profesor, clima de aula, modelo de evaluación; y con el alumno tales como conocimientos previos, habilidades, expectativas y actitudes; 2) Las variables de proceso son las relativas a los efectos de los procesos metacognitivos y de los enfoques de aprendizaje (superficial y profundo) que llevan asociadas; 3) El resultado del aprendizaje está determinado por muchos factores que interactúan entre sí, y pueden ser descritos en términos cuantitativos (cuánto han aprendido), cualitativos (significado de lo aprendido y mejoras cognitivas, actitudinales o procedimentales) o institucionales (calificaciones e indicadores de cambio).

\section{Los estudios relacionados con el proceso de enseñanza-aprendizaje de la Estadística}

En primer lugar, podemos considerar un grupo de estudios centrados en la dimensión afectiva en la que la actitud del alumnado hacia la estadística resulta crucial para un aprendizaje adecuado y uso efectivo de la misma (Carmona, 2004; Estrada, Batanero y Fortuny 2004). Son muchos los trabajos donde se pone de manifiesto la estrecha relación entre la actitud hacia la estadística y el rendimiento académico, entre otros destacamos los de Beins (1985), Katz y Tomezik (1988), Roberts y Saxe (1982), Tempelar et al. (2007) y Wise (1985) y en el ámbito foráneo, así como los de Auzmendi (1992), Gil (1999) y Sánchez-López (1996), Gil (1999), Bayot, Mondéjar, Mondéjar, Monsalve, y Vargas (2005), Mondéjar et al. (2008) en el nacional. En general, todos estos trabajos revelan que la actitud con que el alumnado se enfrenta al aprendizaje de la estadística no es, desde luego, la más adecuada; y ello parece incidir en los resultados de aprendizaje de esta disciplina, de forma que a mejor actitud, mayor rendimiento y viceversa.

Un segundo grupo de investigaciones no menos importantes, es aquel que atiende la forma en que el profesor imparte la clase. En este sentido, destacamos su conocimiento sobre la materia, la forma de comunicarlo, los recursos didácticos utilizados, entre otros. Sobre este 
asunto Bradstreet (1996), propone una colección de estrategias que pueden mejorar la actividad del profesorado, entre otras, establecer unas guías estándar para la buena práctica estadística; asegurarse que el texto guía sea compatible con el nivel de conocimiento de sus lectores; conectar conceptos y métodos estadísticos con un software amigable Behar y Grima (2001); insiste, además, en la importancia de que la enseñanza requiere ser construida sobre la base de experiencias personales de quien está aprendiendo, tratando de conectar conceptos abstractos con experiencias personales, así como desarrollando analogías, símiles y metáforas que pueden ser herramientas instruccionales muy útiles.

Tambien Burril (1990), planteó un conjunto de buenas prácticas que pueden incidir positivamente en la enseñanza de la estadística. En este sentido, habla de que el aprendizaje debe ser interactivo y constructivo, con un papel activo del estudiante; el alumnado debería experimentar primero con estrategias sencillas como el conteo de frecuencias, creación de gráficas sencillas... antes de afrontar aprendizajes más complejos; los temas estadísticos deben estar envueltos por diseños y presentaciones que hagan atractivos los contenidos; el énfasis de cualquier trabajo de estadística debe centrarse, no tanto en la precisión de los resultados, como en la buena interpretación que se haga de ellos. Buscar situaciones problema cercanas a las motivaciones e intereses de los estudiantes ayuda a superar estas fobias.

Un tercer grupo de trabajos, es el que se ocupa de la dedicación del alumnado al aprendizaje de la materia estadística. Relacionado con dicha dimensión están, sin duda, la actitud hacia la disciplina, la motivación con que afronta su aprendizaje y, por tanto, el grado de asistencia a las clases (Rodríguez y Herrera, 2009), así como el grado de utilidad que le confieren (Sahai, Behar y Ojeda, 1998). Si dichos condicionantes adoptan connotaciones negativas pueden ser un motivo corriente para estudiar estadística porque la asignatura aparece en el plan de estudios y es indispensable aprobarla para obtener el título que se pretende. Desafortunadamente, este motivo, generaría aprendizajes de orden superficial de los descritos por Biggs (2005), tipo memorístico, que en poco o nada ayudarían al alumnado a consolidar un conocimiento estadístico profundo y fundamentado. La potenciación de la motivación intrínseca para que sea el propio alumnado el que participe del proceso de aprendizaje apreciando su utilidad puede considerarse una estrategia adecuada.

Así con Behar y Grima (2001, p.196) tareas que signifiquen un reto alcanzable y que involucren una moderada cantidad de discrepancia o aparente incongruencia, estimulan la 
curiosidad del estudiante y se constituyen en un elemento de motivación intrínseca. En definitiva, plantear problemas relacionados con la profesión que difícilmente pudieran ser resueltos sin el uso de la estadística son una excelente forma de motivar a los estudiantes en su estudio.

Finalmente, destacamos un cuarto centro de interés relativo a las herramientas tecnológicas y su influencia en el proceso de enseñanza-aprendizaje de la estadística. En este sentido, podemos afirmar con Mondéjar, Vargas y Mondéjar (2007, p.32) que tanto las nuevas tecnologías, como la educación virtual se están convirtiendo en una de la nuevas opciones más populares, no sólo por ofrecer metodologías de fácil adaptación a una amplia gama de alumnos, sino también por la importancia que asigna a los procesos autodidácticos, a la búsqueda del conocimiento y al fomento de la investigación, así como a la mejora de los procesos de enseñanza-aprendizaje (De la Fuente, Cano, Justicia, Pichardo, García, Martínez y Sander, 2005).

De estas herramientas tecnológicas, Alpízar (2007:101) destaca junto a tutoriales y recursos de Internet y micromundos a los paquetes estadísticos que, según Balacheff y Kaput (1996), permiten la conexión entre la estadística y la vida cotidiana, ya que dan acceso al modelado de situaciones concretas y uso de datos reales. Un ejemplo reciente en esta dirección es la experiencia de Cuesta y Herrero (2008). Efectivamente, la presencia de la tecnología en el aula de estadística se convierte en una herramienta poderosa capaz de aportar a las sesiones de clase sistemas de representación que puedan ser utilizados para la visualización y experimentación de conceptos importantes (Alpízar, 2007, p.98).

Las nuevas tecnologías han cuestionado la manipulación rutinaria de datos con lápiz y papel al erigirse en estrategias más eficaces para resolver problemas con mayor precisión (Santos, 1997:5). Ello facilita la organización y el análisis de los datos y los cálculos se hacen de manera más simple y ordenada permitiendo que el alumnado se libere de procesos de cálculo más o menos farragosos para poder dedicarle más tiempo a la interpretación + discusión + reflexión de los resultados (Ben-Zvi, 2000; Ben-Zvi y Arcavi, 2001; Marshall, Makar y Kazak, 2002). 


\section{Objetivos del estudio}

El objetivo general del estudio es determinar la influencia que una colección de variables relevantes (predictivas), basadas en la opinión del alumnado, juegan en el rendimiento académico de la asignatura de Bases Metodológicas de la Investigación Educativa en el apartado dedicado al análisis cuantitativo de datos (estadística) en la Licenciatura de Pedagogía de la Universidad de Granada.

De manera más precisa, por tanto, el objetivo que guía la presente investigación es determinar la influencia que las variables: opinión del alumnado hacia la estadística, su dedicación a la parte de estadística de la asignatura, labor del docente en la enseñanza de la parte estadística de la asignatura, ayuda de cuadernos de prácticas de SPSS, así como ayuda de otros materiales curriculares introductorios han jugado en la resolución de actividades relacionadas con el análisis de datos cuantitativos y desarrolladas por el alumnado de primero de la Licenciatura de Pedagogía de la Universidad de Granada.

\section{Método}

\section{Participantes}

La población objeto de estudio está referida al alumnado que ha cursado la asignatura de Bases Metodológicas de la Investigación (grupo A) durante el curso académico 2006-07, según datos de matriculación oficial: 117 alumnas/os. No obstante, en la presente investigación no cabe hablar de proceso de muestreo, ya que las/os participantes en la misma ha sido aquellas/os que se presentaron a la convocatoria oficial de junio de 2007 , es decir, un $61,4 \%$ del total. No incidimos en rasgos diferenciales, según algunas variables relevantes como edad, género..., ya que dichas variables cuentan con muy poca variabilidad entre la muestra objeto de estudio con predominio manifiesto de mujeres y edades entre los 18 a 19 años.

\section{Instrumento}

Como instrumento de recogida de datos se utilizó una prueba elaborada ad hoc consistente en la resolución de problemas a partir de un protocolo de 5 situaciones problemáticas 
sobre diferentes contenidos estadísticos de la asignatura. Además el instrumento estaba encabezado por 5 cuestiones adicionales donde se solicitaba al alumnado la valoración de 5 variables diferentes, que han actuado como predictores, mediante una escala en formato Likert que encabezaba la prueba oficial de Bases Metodológicas de la Investigación Educativa de la convocatoria de junio de 2007. Por tanto, los alumnos antes de resolver los problemas planteados debían valorar las cinco variables contempladas con sus correspondientes niveles de respuesta que anteriormente ya hemos explicitado.

\section{Procedimiento}

El presente estudio posee un carácter claramente correlacional-predictivo. Para muchos autores el método correlacional no tiene entidad propia, entre otros Van Dalen y Meyer, (1983). Sin embargo, son más los que sí consideran que posee entidad propia, entre otros Arnal, Del Rincón y Latorre, (1994); Cohen y Manion (2002), Bisquerra (2004) o Etxeberría (1999), ya que dicha metodología va más allá de la mera descripción generando con frecuencia estudios predictivos basados en la regresión (Arnal et al., 1994:184). Con Arnal et al. (1994:188), además, podemos afirmar que la regresión consiste en aproximar los puntos de un diagrama de dispersión a una línea recta con el fin de poder predecir valores a partir de la ecuación de dicha recta o ecuación de regresión. Así pues, se ha pretendido determinar la influencia que un conjunto de variables relevantes de carácter predictivo poseen en el rendimiento académico que alcanza el alumnado en la parte de estadística de la asignatura de Bases Metodológicas de la Investigación Educativa. A continuación explicitamos las variables relevantes consideradas, así como los diferentes niveles contemplados en cada una de ellas.

a) Opinión hacia la estadística con 5 niveles de respuesta: muy mala; mala; aceptable, buena y muy buena.

b) Labor del docente en la transmisión y comprensión de contenidos de estadística de la asignatura con 5 niveles de respuestas: muy mala; mala; aceptable; buena y muy buena.

c) Dedicación del alumnado a la preparación de la parte estadística de la asignatura con 5 niveles de respuesta: muy poca; poca; moderada; mucha y muchísima.

d) Ayuda de los cuadernos de SPSS en la preparación de la parte estadística de la asignatura con 5 niveles de respuesta: muy baja; baja; moderada; alta y muy alta. 
e) Ayuda de otros materiales curriculares de iniciación al análisis cuantitativo en la preparación de la parte estadística de la asignatura con 5 niveles de respuesta: muy baja; baja; moderada; alta y muy alta.

Como variable criterio hemos contemplado la calificación final obtenida por el alumnado en la parte práctica de la prueba desarrollada, es decir, en la de problemas estadísticos planteados. Finalmente, como criterios de calidad del instrumento de medida dedicado a la opinión recolectada en formato Likert, hemos contemplado los parámetros de fiabilidad y validez. Del primero, además, por las características de la administración (1 sola administración), la fiabilidad como consistencia interna. Los resultados logrados por el coeficiente de mayor precisión y uso para este tipo de situaciones, alfa de Cronbach, ha sido el siguiente:

\section{Tabla 3. Alfa de Cronbach del instrumento de recogida de información utilizado}

\begin{tabular}{|c|c|}
\hline $\begin{array}{c}\text { Cronbach's } \\
\text { Alpha }\end{array}$ & N of Items \\
\hline, 878 & 5 \\
\hline
\end{tabular}

Con Morales (2008), podemos afirmar que el coeficiente calculado resulta ser satisfactorio $(\alpha=.87)$, ya que sólo valores por debajo 0.6 pueden considerarse como cuestionables en casos como el que nos ocupa (investigación empírica o general).

Por otra parte, en relación a la validez hemos contemplado la validez de contenido y la de tipo criterial. Para la primera, validez de contenido, hemos verificado que la introducción de las 5 variables contempladas resulta adecuada. Para ello nos basamos en el alfa de Cronbach alcanzado si cada una de las variables no fuese incluida. En la siguiente tabla podemos observar como dicho coeficiente, en todo caso, disminuiría si no las incluyéramos, excepción de ayuda de otros cuadernos, cuya desaparición significaría una leve mejoría del la fiabilidad alcanzada.

En cuanto a la validez criterial hemos correlacionado cada ítem con el total de los 5 (criterio) menos el implicado (correlación corregida) habiéndose obtenido coeficientes de correlación de Pearson moderados y estadísticamente significativos. Ello denota, en todo caso, una validez criterial adecuada. 
Tabla 4. Correlaciones corregidas de cada variable y alfa de Cronbach de la prueba si se elimina la variable indicada

\begin{tabular}{c|c|c}
\hline Variables & $\begin{array}{c}\text { Corrected Item- } \\
\text { Total Correla- } \\
\text { tion }\end{array}$ & $\begin{array}{c}\text { Cronbach's } \\
\text { Alpha if Item } \\
\text { Deleted }\end{array}$ \\
\hline Opinión estadística & $.770^{*}$ & .842 \\
\hline Dedicación asignatura & $.813^{*}$ & .829 \\
\hline Papel profesor & $.826^{*}$ & .828 \\
\hline Ayuda cuadernos SPSS & $.698^{*}$ & .861 \\
\hline Ayuda otros materiales & $.538^{*}$ & .888 \\
\hline Correlaciones estadísticamente significativas con un $\alpha=.01$ bilateral.
\end{tabular}

\section{Análisis estadísticos}

Para el análisis de los datos recogidos en el estudio hemos aplicado un análisis de regresión lineal múltiple que hemos configurado con Cea D'Ancona (2002:65) en torno a diversas etapas: 1) Preámbulos al análisis de regresión múltiple que incluiría: análisis de correlaciones bivariables a partir de la matriz de correlaciones (indagación exploratoria), comprobación de los principales supuestos básicos previos para el análisis de regresión múltiple; 2) Estimación de la ecuación de regresión; 3) Evaluación del modelo resultante.

\section{Resultados}

Preámbulos al análisis de regresión múltiple

Análisis de correlaciones bivariables a partir de la matriz de correlaciones

Antes de acometer el procedimiento de análisis de la regresión múltiple lineal hemos implementado las correlaciones existentes entre la variable criterio y las consideradas independientes. El objetivo de tal procedimiento es doble. Por una parte, sirve para verificar la intensidad de la relación entre la variable criterio y las predictivas de cara poder continuar o no con el procedimiento de análisis. Por otra parte, sirve para establecer a partir de tal intensidad correlacional una especie de ranking de mayor a menor que sirva como criterio de orden para introducirlas en el modelo de regresión que se va determinar. 
Tabla 5. Matriz de correlaciones entre la variable criterio y las predictivas

\begin{tabular}{c|c|c|c|c|c|c}
\hline Variable criterio & $\begin{array}{c}\text { Variables predicti- } \\
\text { vas }\end{array}$ & Opin.estadís. & $\begin{array}{c}\text { Dedic. asignatu- } \\
\text { ra }\end{array}$ & $\begin{array}{c}\text { Actuación del } \\
\text { profesor }\end{array}$ & $\begin{array}{c}\text { Ayuda cuadernos } \\
\text { SPSS }\end{array}$ & $\begin{array}{c}\text { Ayuda de otros } \\
\text { materiales }\end{array}$ \\
\hline \multirow{2}{*}{$\begin{array}{c}\text { CALIFICACIÓN } \\
\text { FINAL }\end{array}$} & $\begin{array}{c}\text { Correlación de Pear- } \\
\text { son }\end{array}$ & $.70\left(^{* *}\right)$ & $.63\left(^{* *}\right)$ & $.703\left(^{* *}\right)$ & $.46\left(^{* *}\right)$ & $.32\left(^{* *}\right)$ \\
\cline { 2 - 7 } & Sig. (bilateral) &, 000 &, 000 &, 000 & .000 & .006 \\
\cline { 2 - 7 } & $\mathrm{N}$ & 70 & 70 & 70 & 70 & 70 \\
\hline
\end{tabular}

** La correlación es significativa al nivel 0,01 (bilateral).

A tenor de los resultados obtenidos en esta primera aproximación exploratoria podemos afirmar, en primer lugar, que las correlaciones resultantes son todas estadísticamente significativas a un nivel $\alpha=.01$ bilateral y que algunas, además, son realmente importantes. En segundo lugar, las correlaciones obtenidas entre la variable criterio con el resto de predictivas ha sido en orden decreciente el siguiente: Actuación del profesor $(r=.703)>$ Opinión previa hacia la estadística $(\mathrm{r}=.70)>$ Dedicación a la asignatura $(\mathrm{r}=.63)>$ Ayuda cuadernos de SPSS $(r=.46)>$ Ayuda de otros materiales curriculares $(r=.32)$. Así pues, ese ha sido el orden en que han sido introducidas en el modelo de regresión lineal múltiple implementado mediante el método por pasos sucesivos.

Comprobación de los principales supuestos básicos previos para el análisis de regresión múltiple

La correcta aplicación del análisis de regresión múltiple exige el cumplimiento de una serie de supuestos previos que informan sobre su conveniencia o descarte. Con Hair, Anderson, Thatam y Black, (1999); Cea D'Ancona (2002) o Etxeberría (1999), entre otros, destacamos sobre todo: la normalidad de las distribuciones de puntuaciones que conforman cada una de las variables implicadas, así como la multicolinealidad entre las mismas.

\section{a) La normalidad}

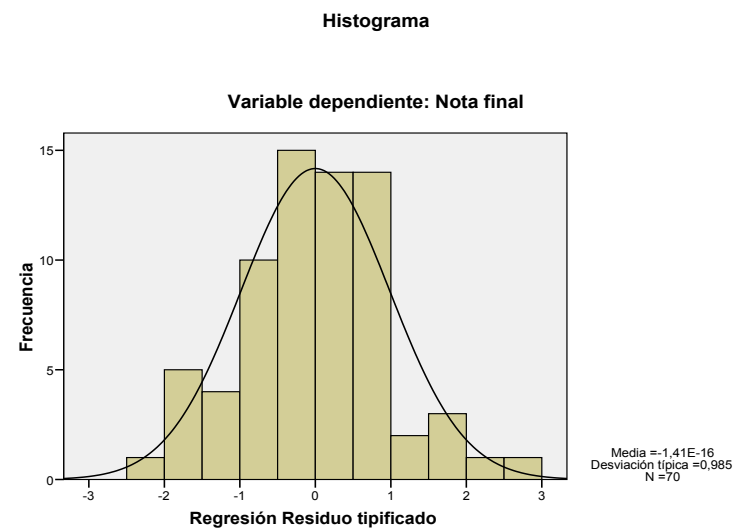

Gráfico 1. Histograma de residuos estandarizados de la variable criterio 
Tabla 6. Índices de asimetría de las variables predictivas contempladas

\begin{tabular}{c|c|c|c|c|c}
\hline Variables predictivas & $\begin{array}{c}\text { Papel } \\
\text { profesor }\end{array}$ & $\begin{array}{c}\text { Opinión } \\
\text { estadística }\end{array}$ & $\begin{array}{c}\text { Dedicación } \\
\text { asignatura }\end{array}$ & $\begin{array}{c}\text { Cuaderno } \\
\text { SPSS }\end{array}$ & $\begin{array}{c}\text { Otros mate- } \\
\text { riales curricu- } \\
\text { lares }\end{array}$ \\
\hline $\mathrm{N}$ Válidos & 70 & 70 & 70 & 70 & 70 \\
\hline Asimetría & .47 & .23 & .16 & .11 & -.26 \\
\hline
\end{tabular}

Como puede apreciarse para la variable criterio el histograma de residuos estandarizados con curva normal denota una normalidad bastante evidente en la que, además, de la forma acampanada simétrica de la curva encontramos valores de distribución con media prácticamente 0 y desviación típica muy próxima a $1 \mathrm{~N}(0,1)$. Por otra parte, para las variables predictivas la asimetría resultante es, fundamentalmente, positiva (exceptuando una variable) con predominio de valores en la parte izquierda de las distribuciones. En todo caso, todos los índices de asimetría por encontrarse dentro del intervalo \pm .5 (Gil, Rodríguez y García, 1995) podrían considerarse casi normales.
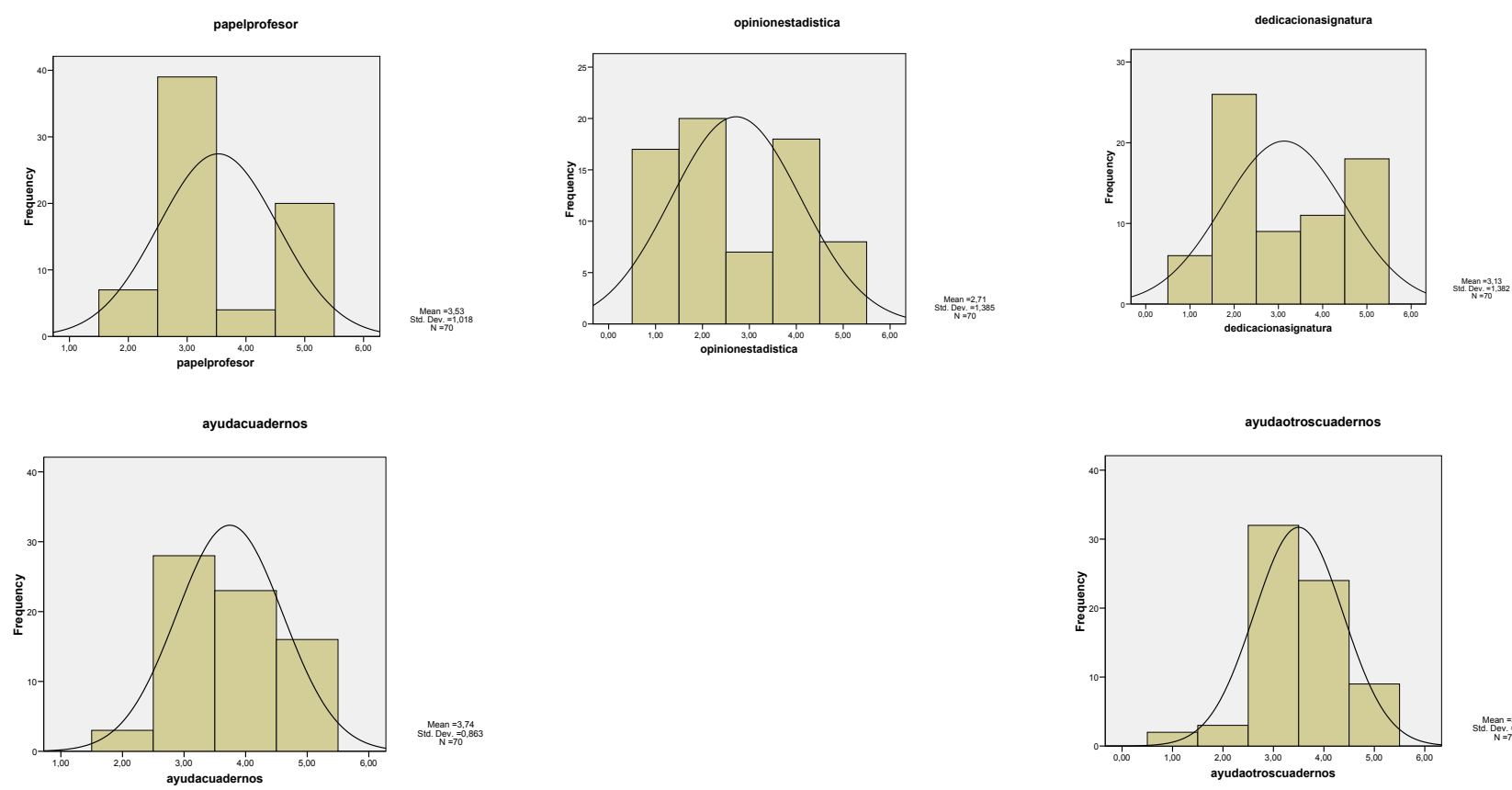

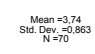

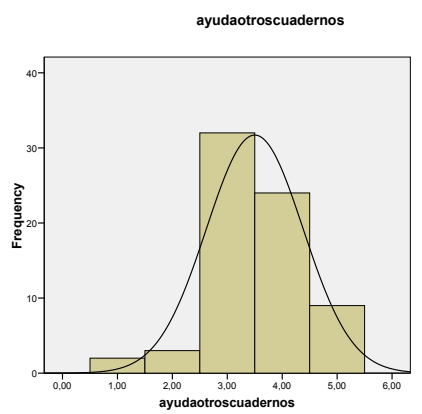

Gráfico 2. Conjunto de histogramas con curva normal referidos a las variables dependientes implicadas 


\section{b) La multicolinealidad}

Se entiende por multicolinealidad aquella situación en la que dos o más variables predictivas del modelo alcanzan correlaciones bivariadas elevadas entre sí lo que puede repercutir negativamente en los resultados que se obtengan del mismo. Para denotar el grado de multicolinealidad de las variables predictivas contempladas se utilizan varias pruebas de diagnóstico, entre las que destacan, el índice de Tolerancia (IT), así como su recíproco o inverso Factor de Inflación de la Varianza (FIV). Para una explicación clara, concisa y fundamentada de ambos pueden consultarse las obras en español de Etxeberria (1999) y Cea D'Ancona (2002). Para nuestro trabajo, por tanto, sólo destacamos las etiquetas interpretativas para ambos. De esta forma, con Etxeberria (1999), Hair et al. (1999) y Cea D'Ancona (2002), podemos afirmar que valores cercanos a la unidad en el caso del IT denotan ausencia casi total de multicolinealidad, razón por lo que valores cercanos a 0 indicaran todo lo contrario. Índices de Tolerancia $($ IT) $<.2$ empiezan a ser preocupantes $\mathrm{y}<.1$ inaceptables. Por su parte, el FIV será mejor cuanto más se acerque a 1 , de tal forma que valores $>5$ se consideran preocupantes y $>10$ intolerables. A continuación explicitamos los resultados obtenidos sólo por las dos variables que han sido consideradas en el modelo de regresión y que con posterioridad apreciaremos con mayor detalle

\section{Tabla 7. Pruebas de colinealidad de las variables predictivas incluidas en la ecuación de regresión}

\begin{tabular}{c|c|c}
\hline Estadísticos de colinealidad & $\begin{array}{c}\text { Tolerancia } \\
\text { (IT) }\end{array}$ & FIV \\
\hline Dedicación asignatura & .496 & 2.016 \\
\hline Opinión estadística & .496 & 2.016 \\
\hline
\end{tabular}

A la luz de los resultados obtenidos podemos afirmar que las variables incluidas en el modelo no adolecen de multicolinealidad, dado están dentro de los límites razonables para que así puedan considerarse. Por tanto, podemos afirmar que dichas variables predictivas, finalmente, incluidas en el modelo de regresión resultan adecuadas para los objetivos predictivos considerados. 
Estimación de la ecuación de regresión

Tabla 8. Proceso de introducción/eliminación de las variables predictivas en el modelo de regresión

\begin{tabular}{c|c|c|c}
\hline Modelo & $\begin{array}{c}\text { Variables intro- } \\
\text { ducidas }\end{array}$ & $\begin{array}{c}\text { Variables } \\
\text { eliminadas }\end{array}$ & Método \\
\hline 1 & $\begin{array}{c}\text { Papel del profe- } \\
\text { sor }\end{array}$ & $\cdot$ & $\begin{array}{c}\text { Por pasos (criterio: Prob. de F para entrar }<=, 050, \\
\text { Prob. de F para salir }>=, 100) .\end{array}$ \\
\hline 2 & $\begin{array}{c}\text { Opinión es- } \\
\text { tadística }\end{array}$ & $\cdot$ & $\begin{array}{c}\text { Por pasos (criterio: Prob. de F para entrar }<=, 050, \\
\text { Prob. de F para salir }>=, 100) .\end{array}$ \\
\hline \multicolumn{2}{|c}{}
\end{tabular}

En estas dos últimas tablas (tabla 8 y 9), podemos apreciar las variables que han sido incluidas en el modelo, finalmente, inferido tras el proceso de selección stepwise y, además, el orden de inclusión de las mismas. En este sentido, se han contemplado dos (papel del profesor y opinión hacia la estadística) y eliminado tres (dedicación a la asignatura, ayuda de cuadernos SPSS, así como ayuda de otros cuadernos).

Tabla 9. Variables excluidas del modelo de regresión finalmente inferido

\begin{tabular}{|c|c|c|c|c|c|c|}
\hline \multicolumn{7}{|c|}{ Variables excluidas ${ }^{c}$} \\
\hline \multirow{2}{*}{\multicolumn{2}{|c|}{ Modelo }} & \multirow[b]{2}{*}{ Beta dentro } & \multirow[b]{2}{*}{$t$} & \multirow[b]{2}{*}{ Sig. } & \multirow{2}{*}{$\begin{array}{l}\text { Correlación } \\
\text { parcial }\end{array}$} & $\begin{array}{l}\text { Estadísticos } \\
\text { de } \\
\text { colinealidad }\end{array}$ \\
\hline & & & & & & Tolerancia \\
\hline \multirow[t]{4}{*}{1} & opinionestadstica &, $382^{\mathrm{a}}$ & 2,953 &, 004 & ,339 & ,396 \\
\hline & dedicacionasignatura &, $168^{\mathrm{a}}$ & 1,104 & ,273 & 134 & 316 \\
\hline & ayudacuadernos &, $087^{a}$ & ,834 & ,407 & 101 & ,675 \\
\hline & ayudaotroscuadernos &, $011^{\mathrm{a}}$ & ,118 & ,906 & 014 & 802 \\
\hline \multirow[t]{3}{*}{2} & dedicacionasig natura &,$- 014^{b}$ &,- 089 & ,930 &,- 011 & ,260 \\
\hline & ayudacuadernos &, $045^{\circ}$ & ,444 & 658 & ,055 & 660 \\
\hline & ayudaotroscuadernos &,$- 011^{b}$ &,- 118 & 907 &,- 015 & ,797 \\
\hline
\end{tabular}

a. Variables predictoras en el modelo: (Constante), papel profesor

b. Variables predictoras en el modelo: (Constante), papelprofesor, opinionestadistica

c. Variable dependiente: Nota final

Tabla 10. Resumen del modelo

\begin{tabular}{c|c|c|c|c}
\hline Modelo & $\mathrm{R}$ & R cuadrado & R cuadrado corregida & Error típ. de la estimación \\
\hline 1 & $.707^{*}$ & .499 & .492 & 1.537 \\
\hline 2 & $.746^{* *}$ & .557 & .544 & 1,456 \\
\hline
\end{tabular}

*Variables predictoras: (Constante), papel profesor

** Variables predictoras: (Constante), papel profesor, opinión estadística

*** Variable dependiente: Nota final 
En la tabla inmediatamente anterior (tabla 10) se explicitan los coeficientes de correlación múltiple, de determinación y determinación corregida, así como los errores típicos de estimación logrados en el modelo inicial y el definitivo. Así pues, podemos afirmar que se ha alcanzado un coeficiente de correlación múltiple de casi .75 y de determinación de .55 , lo que es tanto como decir que la varianza de la variable calificación es explicada en un 55,7\% por las variables papel del profesor y opinión hacia la estadística conjuntamente.

Tabla 11. Componentes de la ecuación de regresión y estadísticos complementarios

\begin{tabular}{c|c|c|c|c|c|c}
\hline \multirow{2}{*}{ Modelo } & & \multicolumn{2}{|c|}{$\begin{array}{c}\text { Coeficientes no } \\
\text { estandarizados }\end{array}$} & $\begin{array}{c}\text { Coeficientes } \\
\text { estandarizados }\end{array}$ & $\mathrm{t}$ & Sig. \\
\hline \multirow{2}{*}{1} & $\mathrm{~B}$ & Error típ. & Beta & & \\
\hline \multirow{2}{*}{2} & (Constante) & -.364 & .668 & & -.546 & .587 \\
\cline { 2 - 8 } & Papel del profesor & 1.498 & .182 & .707 & 8.236 & .000 \\
\cline { 2 - 8 } & Papel del profesor & .869 & .274 & .410 & .362 & .718 \\
\cline { 2 - 8 } & $\begin{array}{c}\text { Opinión estadísti- } \\
\text { ca }\end{array}$ & .595 & .201 & .382 & 2.953 & .002 \\
\hline \multirow{2}{*}{ Variable dependiente: calificación final } & & & & & \\
\hline
\end{tabular}

Como puede apreciarse después de inferirse un primer modelo en el que sólo estaba la variable dedicación a la asignatura se ha propuesto un segundo y definitivo en el que se ha incluido también a la variable opinión hacia la estadística. Por tanto, la ecuación de regresión calculada tendría esta notación estadística:

$Y=\alpha+\beta_{1} x_{1}+\beta_{2} x_{2}+e$

$Y=.241+.869_{\text {actuación del profesor }}+.595_{\text {op.estad. }}+1.45^{*} \mathrm{z \alpha}$

Otro aspecto importante del modelo inferido es verificar como cada una de las variables predictivas incluidas en el mismo han resultado, por separado, estadísticamente significativas ( $\mathrm{p} \leq 0.05$ mediante pruebas $\mathrm{t}$ ). Dicho aspecto ha quedado refrendado en la tabla 9 y es un indicador más de la bondad del modelo inferido.

\section{Evaluación del modelo}

Para la evaluación del modelo se pueden tomar en consideración distintos criterios. Con Etxeberria (1999) y Cea D'Ancona (2002) destacamos entre los fundamentales: el coeficiente de determinación $\left(\mathrm{R}^{2}\right)$, los gráficos sobre el ajuste a la recta de regresión, así como la significatividad del modelo medida a través del análisis de la varianza (ANOVA). 
Del coeficiente de determinación corregido hemos comentado ya que asciende a .544, lo que equivale a afirmar que un porcentaje importante de la $S^{2}$ de la variable criterio (calificación), casi un $55 \%$, es explicada por las variables predictivas incluidas, finalmente, en el modelo (papel del profesor y opinión hacia la estadística). En cuanto a los gráficos sobre el ajuste del modelo proponemos el siguiente:

\section{Gráfico de dispersión}

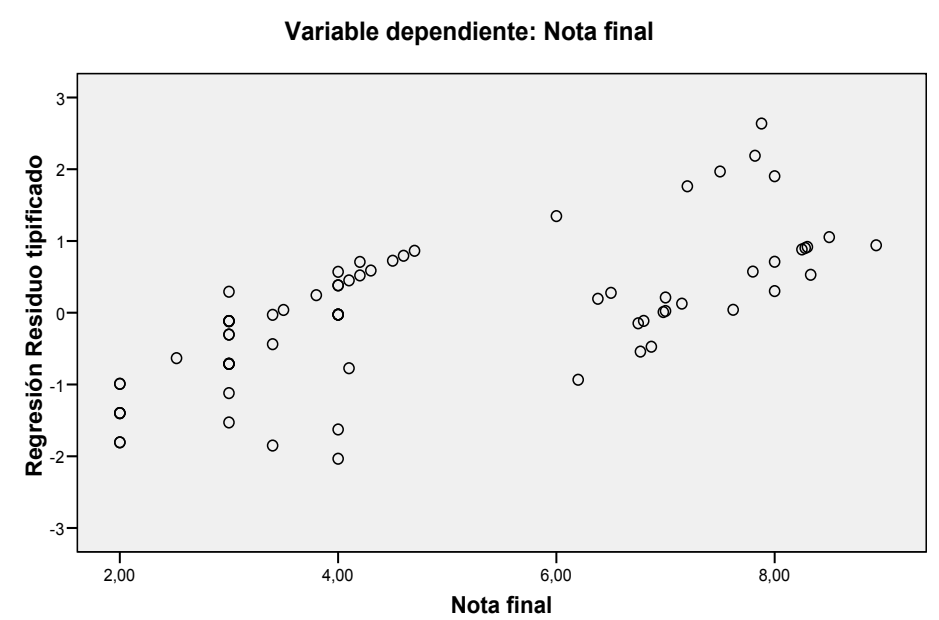

\section{Gráfico 3. Gráfico de dispersión sobre el ajuste del modelo}

En el mismo puede apreciarse un ajuste más o menos moderado entre los valores reales y pronosticados por el modelo debido a que los pares de valores se sitúan en mayor o menor medida entorno a la línea imaginaria conformada por la recta de regresión.

Finalmente, se trata de comprobar si el efecto conjunto de las dos variables predictivas que han demostrado ser relevantes en la predicción de la variable criterio en el modelo 2 (modelo definitivo) difiere estadísticamente de cero o no. Para ello se ha implementado el siguiente $A N O V A$ :

Tabla 12. Anova asociado al modelo de regresión ANOVA

\begin{tabular}{c|c|c|c|c|c|c}
\hline \multirow{4}{*}{ Modelo } & $\begin{array}{c}\text { Fuentes de varia- } \\
\text { ción }\end{array}$ & Suma de cuadrados & $\mathrm{gl}$ & $\begin{array}{c}\text { Media } \\
\text { cuadrática }\end{array}$ & $\mathrm{F}$ & Sig. \\
\hline \multirow{3}{*}{1} & Regresión & 160,348 & 1 & 160,348 & 67,834 &, $000^{*}$ \\
\cline { 2 - 7 } & Residual & 160,741 & 68 & 2,364 & & \\
\cline { 2 - 7 } & Total & 321,088 & 69 & & & \\
\hline \multirow{2}{*}{2} & Regresión & 178,861 & 2 & 89,431 & 42,129 &, $000^{* *}$ \\
\cline { 2 - 7 } & Residual & 142,227 & 67 & 2,123 & & \\
\cline { 2 - 7 } & Total & 321,088 & 69 & & & \\
\hline
\end{tabular}

* Variables predictoras: (Constante), papel profesor ** Variables predictoras: (Constante), papel profesor, opinión estadística *** Variable dependiente: Nota final 
Efectivamente, se ha alcanzado una razón $\mathrm{F}$ importante asociada a una $\mathrm{p}=, 000$. Este dato nos indica que el modelo de regresión inferido adquiere significatividad estadística, es decir, que la variación explicada es más importante que la no explicada y resulta, por tanto, relevante para los objetivos predictivos para los que ha sido desarrollado.

\section{Discusión y Conclusiones}

La enseñanza-aprendizaje de la Estadística constituye una competencia básica de los titulados en el campo de las Ciencias Sociales. Hay evidencia de que no es de las asignaturas que despierten mayores dosis de motivación por parte de los universitarios; tampoco los rendimientos arrojan las mejores tasas de éxito. Investigar los procesos que condicionan la adquisición de estas competencias, determinar las variables que influyen de manera directa e indirecta y analizar los grupos de factores que condicionan en mayor medida el éxito de los aprendizajes ha de permitir optimizar la enseñanza de esta disciplina tan denostada.

A lo largo de las últimas décadas, la investigación educativa ha puesto énfasis en la exploración de variables motivacionales, contextuales, cognitivas y académicas; también bastantes trabajos han abierto nuevos frentes de exploración innovadora basados en el aprendizaje basado en problemas (ABP) o en estudios de corte más procedimental centrados en la adquisición de competencia tecnológica, comunicación de lenguajes apropiados, interpretación de resultados y análisis de datos.

El encuadre global de las aproximaciones ha permitido abordar parcelas desconectadas de grupos de variables aisladas, se echa en falta, en consecuencia, la búsqueda de estructuras de fundamentación integradas y análisis empíricos que aterricen en visiones comprensivas que analicen la interacción entre estos grupos de variables.

Volviendo a los resultados obtenidos en el presente estudio se han obtenido correlaciones significativas de la variable criterio rendimiento académico en la asignatura de Bases Metodológicas de la Investigación Educativa con cinco grupos de variables predictivas orientadas hacia un modelo de enseñanza-aprendizaje profundo en el que, tanto el estudiante, como el profesor mantienen un nivel de compromiso, motivación y exigencia con la materia que se identifica con los rasgos señalados en la literatura que definen un modelo de trabajo universitario activo y motivador. 
Los cinco grupos de variables elegidos son: papel del profesor $(\mathrm{r}=0,703)$, opinión hacia la estadística $(\mathrm{r}=0,70)$, dedicación a la asignatura $(\mathrm{r}=0,63)$, ayuda de cuadernos de apoyo a la docencia $(\mathrm{r}=0,46)$ y ayuda de otros materiales docentes $(\mathrm{r}=0,32)$. El análisis exploratorio de las ecuaciones de regresión que mejor predicen el rendimiento académico de los estudiantes, previa verificación del cumplimiento de los supuestos de normalidad y multicolinealidad pone de manifiesto que las variables que mejor ejercen de predictoras en este modelo son el papel del profesor y la opinión previa que los estudiantes poseen sobre la estadística; el resto de variables no resultan determinantes en el modelo, aunque, como después explicitamos que otra situación de estudio reflejase diferente configuración. De cualquier forma, el modelo inferido permite explicar casi un 55\% de la varianza de los resultados del rendimiento con estas dos variables predictoras, con un nivel de significación muy por debajo del 0.05.

En consecuencia y una vez examinados los resultados obtenidos y a falta de futuras aproximaciones, probablemente de mayor complejidad (análisis causal), que confirmen esta tendencia es posible aventurar que es necesario trabajar con el alumnado su actitud previa hacia la estadística, con el objetivo de polarizarla hacia posiciones más positivas, a la vez que reflexionar entorno a la labor docente en la transmisión de este tipo de contenidos estadísticos en aspectos tales como: lenguaje inteligible para el alumnado, ejemplos cercanos y reales, desarrollos atractivos y motivantes de los contenidos, importancia de la competencia argumentativa en detrimento de los desarrollos estadísticos-matemáticos, aprendizaje constructivo, entre otros. En definitiva hacia aspectos claramente posicionados en un estilo de aprendizaje profundo. 


\section{Referencias}

Alpízar, M. (2007). Herramientas tecnológicas en el proceso de enseñanza-aprendizaje de la estadística. Cuadernos de Investigación y Formación en Educación Matemática, 2(3), 99-118.

Arnal, J.; Del Rincón, D. y Latorre, A. (1994). Investigación educativa. Fundamentos y metodología. Barcelona: Labor.

Auzmendi, E. (1992). Las actitudes hacia la matemática-estadística en las enseñanzas medias y universitarias. Bilbao: Mensajero.

Balacheff, N. y Kaput, J.J. (1996). Computer-based learning environments in mathematics. En Bishop, A.J.; Clements, K.; Keitel, C; Kilpatrick, J. y Laborde, C. (Eds.) International Hanbook of Mathematics Education (pp. 469-501). Netherlands: Kluwer Academic Publishers.

Bayot, A., Mondéjar, J., Mondéjar, J.A., Monsalve, F. y Vargas, M. (2005). The Difficulties of Learning Concepts in the Social Sciences. En Misztal, M. and M. Trawinski (eds.) Studies in Teacher Education: Psichopedagogy (pp. 242-258). Wydawnictwo Naukowe Akademii Pedagogicznej: Kraków.

Behar, R. y Grima, P. (2001). Mil y una dimensiones del aprendizaje de la estadística. Estadística Española, 43(148), 189-207.

Beins, B.C. (1985). Teaching the relevance of statistics trough consumer-oriented research. Teaching of Psychology, 12, 168-169.

Ben-Zvi, D. (2000). Toward understanding the role of technological tools in statistical learning. Mathematical Thinking and Learning, 2(1-2), 127-155.

Ben-Zvi, D. y Arcavi, A. (2001). Junior high school students construction of global views of data an data representations. Educational Studies in Mathematics, 45 (1-3), 35-65.

Biggs, J. (2005). Calidad del aprendizaje universitario. Madrid: Narcea.

Bisquerra, R. (2004). Metodología de la investigación educativa. Madrid: La Muralla.

Bradstreet, T.E. (1996). Teaching Introductory Statistics Courses So that Non statisticians Experience Statistical Reasoning. The American Statsitician. 50(1), 57-71.

Burril, G. (1990). Quantitative litaracy. Leadership training for masters teachers. En Hawkins, A. (Ed.). Training tearchers to teach statistics. (pp. 219-227). The Netherlands: International Statistical Institute, UNESCO. 
Carmona, J. (2004). Una revisión de las evidencias de fiabilidad y validez de los cuestionarios de actitudes y ansiedad hacia laestadística. Statistics Education Research Journal, 3(1), 5-28. En: http://www.stat.auckland.ac.nz/serj

Cea D’Ancona, M.A. (2002). Análisis multivariante. Teoría y práctica en la investigación social. Madrid: Síntesis.

Cohen, L. y Manion, L. (2002). Métodos de investigación educativa. Madrid: La Muralla.

Cuesta, M. y Herrero, J. (2008). Curso 0 de análisis de datos en Psicología. Tutorial Hipermedia. Electronic Journal of Research in Educational Psychology, 16, 6(3), 847-862.

De la Fuente, J., Cano, F., Justicia, F., Pichardo, M.C., García, A.B., Martínez, J.M., y Sander, P. (2005). Efectos de la utilización de herramientas on-line en la mejora de la regulación del proceso de enseñanza-aprendizaje. Electronic Journal of Research in Educational Psychology, 13, 5(3), 757-782.

De la Fuente, J. y Justicia, F. (2007). El modelo DIDEPRO de regulación de la enseñanzaaprendizaje: avances recientes. Electronic Journal of research in Educational Psycho$\log y 13,5(3), 535-564$.

Dillon, K. M. (1982). Statisticophobia. Teaching of Psychology, 9(2), 117-131.

De Miguel, M. (Coord.) (2006). Metodologías de enseñanza y aprendizaje para el desarrollo de competencias. Orientaciones para el profesorado ante el Espacio Europeo de Educación Superior. Madrid: Alianza Editorial.

Estrada, A., Batanero, C. y Fortuny, J. M. (2004). Un estudio comparado de las actitudes hacia la estadística en profesores en formación y en ejercicio. Enseñanza de las Ciencias, 22(2), 263-274.

Etxeberría, J. (1999). Regresión Múltiple. Madrid: La Muralla.

Evans, C.J., Kirby, J. R. y Fabrigar, L.R. (2003). Approaches to learning, need for cognition, and strategic flexibility among university students, British Journal of Educational Psychology, 73, 507-228.

Gil, J. (1999). Actitudes hacia la estadística. Incidencia de las variables sexo y formación previa. Revista española de pedagogía, 57(214), 567-590.

Gil, J., Rodríguez, G., y García, E. (1995). Estadística Básica Aplicada a las Ciencias de la Educación. Sevilla: Kronos.

Gordon, S. (1995). A theoretical Approach to Understanding Learners of Statistics. Journal of Statistical Education, 3(3), 45-57.

Hair, J.F., Anderson; R.E., Thatam, R.L. y Black, W.C. (1999). Análisis multivariante. Madrid. Prentice Hall. 
Hernández, F., Martínez, P., Da Fonseca, P.S y Rubio, M. (2005). Aprendizaje, competencias y rendimiento en Educación Superior. Madrid: La Muralla.

Justicia, F., Pichardo M.C., Cano F., García A.B., De la Fuente, J. (2008). The Revised TwoFactor Study Process Questionnaire (R-SPQ-2F): Exploratory and confirmatory factor analyses at item level. European Journal of Psychology of Education, 23(1), 355-372.

Katz, B. M. y Tomazic, T. Z. (1988). Changing student's attitudes toward statistics through a nonquantitative approach. Psichological Reports, 62, 658.

Marshall, J., Makar, K., y Kazak, S. (2002). Young urban students conceptions of data uses, representation and analysis. OPME-MA XXIV, Athens, Georgia, 1292-1304.

Mondéjar, J., Vargas, M., y Mondéjar, J.A. (2007). Impacto del uso del e-learning en las actitudes hacia la estadística. Revista Latinoamericana de Tecnología Educativa, 6(2), 3147.

Mondéjar, J., Vargas, M. y Bayot, A. (2008). Medición de la actitud hacia la estadística. Influencia de los procesos de estudio. Electronic Journal of Research in Educational Psychology, 16, 6(3), 729-748.

Morales, P. (2008). Estadística aplicada a las Ciencias Sociales.Madrid: Universidad Pontificia Comillas.

Onwuegbuzie, A. J., y Wilson, V. A. (2003). Statistics anxiety: nature, etiology, antecedents, effectsand treatments-a comprehensive review of the literature. Teaching in Higher Education, 8(2), 195-209.

Pérez, A. (1983). Paradigmas contemporáneos de investigación didáctica. En Gimeno, J. y Pérez, A.: La enseñanza: su teoría y su práctica (pp. 95-138). Madrid: Akal.

Perney, J., y Ravid, R. (1990, Abril). The relationship between attitudes towards statistics, math selfconcept, test anxiety and graduate students' achievement in an introductory statistics course. Comunicación presentada al Annual Meeting of the American Educational Research Association,Boston, MA, EE. UU.

Roberts, D. M. y Saxe, J. E. (1982). Validity of a statistics attitude survey: a follow-up study. Educational and Psichological Measurement, 42, 907-912.

Rodríguez, C. y Herrera, L. (2009). Análisis correlacional-predictivo de la influencia de la asistencia a clase en el rendimiento académico universitario. Estudio de caso de una asignatura. Profesorado, Revista de Currículum y Formación del Profesorado, 13(2), $1-14$.

Sahai, H., Behar, R. y Ojeda, M.M. (1998). Un replanteamiento del problema de la educación estadística: perspectiva desde el aprendizaje. Biometrical Education: problems, ex- 
periences and solutions. International Biometric Society Networkfor Central American and Caribean. San José. Costa Rica.

Sánchez-López, C.R. (1996). Validación y análisis ipsativo de la escala de actitudes hacia la estadística (EAE). Análisis y Modificación de Conducta, 22(86), 799-819.

Santos, M. (1997). Principios y métodos de la resolución de problemas en el aprendizaje de las matemáticas. México: Grupo Editorial Iberoamericano.

Tempelaar, D. T., Van der Loeff, S. S., y Gijselaers, W. (2007). A structural equation model analyzing the relationship of students' attitudes toward statistics, prior reasoning abilities and course performance. Statistics Education Research Journal, 6(2), 78-102.

Van Dalen, D.B. y Meyer, W.J. (1983). Manual de técnicas de investigación educacional. Buenos Aires: Paidós.

Waugh, R. F. (2002). Measuring self-reported studying and learning for university students: linking attitudes and behaviours on the same scale. British Journal of Educational Psychology, 72, 573-604.

Wise, S. (1985). The development and validation of a scale measuring attitudes toward statistics. Educational and Psychological Measurement, 45, 401-405. 
[Página en blanco por razones de paginación] 